\title{
HÁBITOS DE ALIMENTACIÓN SALUDABLE EN ESTUDIANTES UNIVERSITARIOS
}

\author{
HEALTHY EATING HABITS IN COLLEGE STUDENTS
}

Juana María Saucedo-Soto ${ }^{1}$ * (D); Alicia del Socorro de la Peña de León ${ }^{2}$ (D); Juan Bernardo Amezcua-Núñez ${ }^{3}$ (D).

1.Universidad Autónoma de Coahuila, México.juanasaucedosoto@uadec.edu.mx

2.Universidad Autónoma de Coahuila, México. aliciadelapenadeleon@uadec.edu.mx

3.Universidad Autónoma de Coahuila, México.juan.amezcua@uadec.edu.mx

*Autor de Correspondencia: Juana María Saucedo-Soto, correo electrónico: juanasaucedosoto@uadec.edu.mx

\section{RESUMEN}

Una alimentación saludable es determinante para el buen estado de salud de las personas en cualquier etapa de su ciclo vital. Esta alimentación debe contener los nutrientes esenciales, además de ser variada y adecuada a la edad, peso, talla, sexo, condición física de cada persona. El presente estudio se plantea como objetivo identificar los hábitos de alimentación saludable en estudiantes universitarios, así como conocer si existió cambio en sus hábitos a raíz de la pandemia de Covid-19. Se recolectaron datos de 408 estudiantes, a través de un instrumento de medición estructurado, el test Kidmed. Los estadísticos utilizados fueron pruebas no paramétricas: Mann-Withney y Kruskal-Wallis. Los resultados obtenidos fueron: los estudiantes presentaron una alimentación media, y se necesita mejorar los hábitos alimenticios. Se encontró diferencias de género, las mujeres presentan un mayor consumo de frutas, verduras, arroz y pescado que los hombres, en cuanto a la edad los hábitos positivos como son: consumir fruta, verduras, pescado, legumbres, frutos secos, predominan los estudiantes en un rango de edad de 20 a 23 y mayores de 25 años. El $54.53 \%$ de los estudiantes tienen hábitos negativos que incluye: consumir comida rápida, no desayunar, o desayunar bollería industrial y consumir golosinas.

Palabras clave: Alimentación saludable; prueba Kidmed; estudiantes universitarios.

Cómo citar:

Saucedo-Soto, Juana María; del Socorro de la Peña de León, Alicia; Amezcua-Núñez, Juan Bernardo. (2021). Hábitos de alimentación saludable en estudiantes universitarios. Revista de Investigaciones Universidad del Quindio, 33(S1), 199-211. https://doi.org/10.33975/ riuq.vol33nS1.492 


\begin{abstract}
A healthy diet is a determining factor for the good health of people at any stage of their life cycle. This diet should contain essential nutrients, in addition to being varied and appropriate to the age, weight, height, sex, and physical condition of each person. The purpose of this research is to identify the healthy eating habits of university students, as well as to determine whether there was a change in their habits as a result of the Covid-19 pandemic. Data were collected from 408 students through a structured measurement instrument, the Kidmed test. The statistics used were nonparametric tests: Mann-Withney and Kruskal-Wallis. The results obtained were: the students presented an average diet and there is a need to improve eating habits. Gender differences were found, women presented a higher consumption of fruits, vegetables, rice and fish than men, in terms of age the positive habits such as: consume fruit, vegetables, legumes, nuts, predominate students in an age range of 20 to 23 and older than 25 years. The $54.53 \%$ of the students have negative habits that include: eating fast food, not eating breakfast, or eating industrial pastries for breakfast and consuming sweets.
\end{abstract}

Keywords: Healthy eating; Kidmed test; college students.

\title{
INTRODUCCIÓN
}

De acuerdo a la OMS (2018) llevar una dieta sana a lo largo de la vida ayuda a prevenir la malnutrición en todas sus formas, así como diferentes enfermedades no transmisibles y trastornos. Una alimentación saludable nos protege de múltiples enfermedades, en especial de las enfermedades no trasmisibles como la diabetes, hipertensión, obesidad y sobrepeso; de allí la importancia que los buenos hábitos alimentarios se inicien en los primeros años de vida y continúen en la vida adulta para mantener la salud y calidad de vida.

La OMS (2015) recomienda una dieta basada en el consumo de frutas, verduras y legumbres, menos del 10\% en consumo de azucares libres, menos del 30\% del consumo de grasas y recomienda las grasas no saturadas que se encuentran en el pescado, aguacate, frutos secos y aceite de oliva y menos de $5 \mathrm{~g}$ de sal.

Una alimentación saludable debe ser variada y equilibrada, la cual nos ayuda a conservar buena salud y nos mantiene protegidos de numerosas enfermedades, esta deberá estar de acuerdo a la edad, peso, talla, sexo, condición física y situación de salud de cada persona. Sin embargo, el aumento de la producción de alimentos procesados, la rápida urbanización y el cambio en los estilos de vida han dado lugar a un cambio en los hábitos alimenticios. La OMS (2015) sostiene que el estilo de vida se basa en la interrelación de sus condiciones y patrones de conducta de las personas, entre ellos la alimentación.

Un segmento importante de estudio son los jóvenes universitarios ya que numerosos estudios han informado que la población estudiantil es un grupo particularmente vulnerable desde el punto de vista nutricional, debido a que se caracteriza por saltarse comidas con frecuencia y picar entre las comidas. (Pi, Vidal Brassesco, Viola, Aballay 2015, p 1748). La vida universitaria es una etapa que se caracteriza por grandes cambios en su estilo de vida. Los jóvenes son un grupo de riesgo que se caracteriza por un estilo de vida poco saludable cargado de estrés y falta de tiempo, lo que conduce al 
consumo de una dieta rápida y poco nutritiva. (Navarro, González, Montero, López, Schmidt, 2015, p. 2651).

El presente estudio se plantea como objetivo identificar los hábitos de alimentación saludable en estudiantes universitarios, así como conocer si existió cambio en sus hábitos a raíz de la pandemia de Covid-19

\section{MARCO TEÓRICO}

Se puede decir que la alimentación saludable es aquella que proporciona los nutrientes que el cuerpo necesita para mantener el buen funcionamiento del organismo, conservar o restablecer la salud, minimizar el riesgo de enfermedades, garantizar la reproducción, gestación, lactancia, desarrollo y crecimiento adecuado. Para lograrlo, es necesario el consumo diario de frutas, verduras, cereales integrales, legumbres, leche, carnes de aves y pescado y aceite vegetal en cantidades adecuadas y variadas.

En el estudio realizado por Troncoso, Doepking y Zuñiga (2013, p. 44) asocia el concepto de "alimentación saludable" al consumo de una dieta balanceada, entendiendo este último concepto como una alimentación que presente un bajo aporte de alimentos de alta densidad energética y una alta concentración de fibra, identificando claramente la importancia de la ingesta de frutas y verduras en la dieta habitual, además de agua.

Sabemos que la educación nutricional debe hacerse en edades tempranas de la vida, la infancia y adolescencia son etapas de desarrollo físico y psicológico, donde se incorporan hábitos, entre ellos los dietéticos, que todos juntos conformaran la calidad de la vida adulta. (Sánchez, Aguilar, González, Esquius, Vaqué, 2017, p. 19)

Las edades tempranas son ideales para inculcar un estilo de vida saludable. Los hábitos alimentarios se forman y desarrollan en la infancia, principalmente en el ámbito familiar. Los conocimientos de los padres y las creencias de éstos sobre lo que alimenta o no, se transmiten desde los primeros años de vida y configuran, en mayor o menor medida, el comportamiento alimentario futuro de un hijo. Igual que es necesario educar en valores, también lo es educar en hábitos alimenticios saludables.

Si una familia tiene buenos hábitos alimenticios, estos repercutirán positivamente en la vida adulta de sus hijos, pero estas buenas costumbres se pueden ver afectadas por diferentes aspectos como son: factores económicos, es más caro comprar productos orgánicos, por modas, creencias, publicidad, influencia social sobre la compra de ciertos productos y todo esto hace que vayamos modificando patrones de comportamiento sobre qué tipo de alimentos consumimos.

Vivimos en una época en la que muchos de los alimentos que consumimos pasan por distintos procesos químicos, son productos industrializados que se venden en grandes cantidades para maximizar su rentabilidad.

Según la WHO (World Health Organization, 2018) las modificaciones en los patrones de consumo de alimentos hacia la preferencia por los alimentos industrializados modernos son la principal causa del dramático incremento de las enfermedades no transmisibles por lo que está muy bien establecido el papel de las dietas y de los patrones de consumo de alimentos como elemento fundamental para 
prevenir esas enfermedades. Si bien la carga genética podría explicar casos familiares o individuales, y la falta de actividad física, la causa primordial de las repercusiones del cambio de estilo de vida son los alimentos industrializados modernos. Esto se ha planteado desde hace algún tiempo, tanto en la prevención y manejo de enfermedades metabólicas y el cáncer. Mencionado por Muñoz, Córdova, Del Valle (2015, p. 1582).

Debido al gran incremento en enfermedades no trasmisibles como la diabetes, hipertensión, obesidad y sobrepeso, organizaciones como la OMS (Organización Mundial de la Salud), WHO (World Health Organization) y la FINUT (Fundación Iberoamericana de Nutrición) hacen propuestas y realizan planes para mejorar la salud de la población a nivel mundial.

Dentro de las propuestas que se ven a diario en páginas de internet se encuentran los diferentes tipos de dietas que buscan mejorar la salud de grupos específicos de personas de acuerdo a sus objetivos.

Según la FINUT (2018) el término dieta se refiere al hábito alimenticio de una persona. Existen una gran variedad de dietas, algunas de ellas hacen referencia simplemente a un determinado estilo de vida, como la dieta mediterránea o la dieta vegetariana. Las dietas terapéuticas están diseñadas para personas con determinadas dolencias, como la dieta libre de gluten para la enfermedad celíaca o la dieta baja en sodio para las personas que sufren hipertensión.

La clasificación de dietas que hace la FINUT son:

Dieta vegetariana. Las dietas vegetarianas se enfocan principalmente en el consumo de productos de origen vegetal (frutas, verduras, legumbres, hortalizas, semillas, granos, etc.). Dieta mediterránea. Se trata de una dieta inspirada en los alimentos que se consumen habitualmente en los pueblos que se encuentran sobre la costa del Mar Mediterráneo. Se puede decir que es una dieta saludable, basada principalmente en pescados, frutas, vegetales, granos integrales, frutos secos y aceite de oliva. Además, existen otro tipo de dietas que son para condiciones de salud específicas como: Dieta baja en sodio, para el tratamiento de la hipertensión arterial. Dieta libre de gluten, para el tratamiento de la enfermedad celíaca. Dieta baja en purinas, para evitar niveles elevados de ácido úrico en sangre. También existen las dietas para adelgazar, en esta clase de dietas hay infinidad de variantes, pero se puede decir que básicamente las dietas para adelgazar se subdividen en:

- Dietas bajas en hidratos de carbono.

- Dietas bajas en grasas.

- Dietas bajas en calorías.

- Dietas proteicas, bajas en hidratos de carbono.

Existen otras que también son importantes como: La Dieta de la Zona. Dieta Vegana. Dieta Orgánica. Esto sustenta que las dietas tradicionales como la mediterránea, la japonesa o la mexicana, no solo tienen valor cultural sino también son biológicamente necesarias para los grupos humanos. (Muñoz et.al., 2015, p.1583)

Segmento de estudiantes universitarios

La vida universitaria es una etapa que se caracteriza por grandes cambios en su estilo de vida. Se han realizado diversos estudios sobre la alimentación que tienen los estudiantes universitarios, debido a 
que son un grupo vulnerable por los horarios de la escuela que pueden ser por la mañana, tarde y/o noche, a eso se le agrega que algunos ya trabajan y tienen que combinar ambas actividades, por lo tanto sus tiempos para comer se reducen y tratan de satisfacer la necesidad de alimento consumiendo comida rápida como, hamburguesas, burritos, etc., o comida chatarra, como frituras, refrescos, pan o galletas.

Un segmento importante de estudio son los jóvenes universitarios ya que numerosos estudios han informado que la población estudiantil es un grupo particularmente vulnerable desde el punto de vista nutricional, debido a que se caracteriza por saltarse comidas con frecuencia y picar entre las comidas. (Pi, et.al., 2015, p 1748). La vida universitaria es una etapa que se caracteriza por grandes cambios en su estilo de vida. Los jóvenes son un grupo de riesgo que se caracteriza por un estilo de vida poco saludable cargado de estrés y falta de tiempo, lo que conduce al consumo de una dieta rápida y poco nutritiva. (Navarro, et.al., 2015, p 2651).

En el estudio realizado por Troncoso et al., los estudiantes identificaron algunas motivaciones como "dificultades para realizar una dieta sana: la facilidad de conseguir en el establecimiento educacional o en sus alrededores, alimentos de bajo costo que presentan alta densidad energética; las preferencias personales de los estudiantes, en donde se privilegia el servicio rápido que presentan los alimentos con una alto aporte de grasas, en demerito de otros como frutas y verduras; la falta de disponibilidad de tiempo durante la jornada académica que facilita la adquisición de alimentos de alta densidad energética en la universidad o a no realizar algunos horarios de alimentación, como el desayuno o almuerzo". (2013, p.44)

Estos factores como la facilidad de comprar en los alrededores de la universidad comida rápida, de bajo costo y la falta de tiempo, los induce a no consumir alimentos saludables como serían frutas y verduras, a picar comida chatarra o simplemente saltarse algunos alimentos como el desayuno.

En el estudio de Troncoso et al., mencionan un ejemplo de respuestas dadas por los estudiantes entrevistados "No, porque a mi igual me gusta la comida chatarra y por los tiempos porque cuando uno tiene 10 minutos no alcanza a comer o en el almuerzo no alcanza a comer algo saludable, como por ejemplo legumbres. Siempre se compra lo más fácil o al comprarse un paquete de papas fritas o una bebida, no te cubren el hambre, sino que uno come algo que te quitan el hambre y que sean cosas ricas". (2013, p.44).

En términos generales se podría suponer que los hábitos alimenticios saludables, dependen de los conocimientos que se tengan sobre alimentación y salud y como consecuencia lógica que a mayor conocimiento mejor alimentación. Sin embargo, de acuerdo a un estudio realizado en estudiantes de medicina en una universidad chilena, concluye que existe una elevada prevalencia de factores de riesgo nutricional, sobrepeso, inactividad y tabaquismo a pesar de los conocimientos que ellos tengan sobre salud. Mencionado por Reyes y Oyola, (2020, p.68).

En una universidad privada se encontró que el 53,4\% tiene un nivel regular de conocimientos sobre alimentación saludable, estos porcentajes varían con el sexo y la edad. Sólo tienen los conocimientos que se les brindó en el hogar. Respecto al sexo, el mayor conocimiento se da en mujeres $(48,4 \%)$ probablemente se debe a que tienen mayor información sobre los alimentos que compran y consumen. (Reyes y Oyola, 2020, p.68). 
El consumir alimentos saludables no solo depende del conocimiento que se tenga porque entran en juego otros factores que ya se mencionaron, el poco tiempo disponible entre clases y/o trabajo, la facilidad de comprar comida rápida cercana a la universidad, el bajo costo de la misma, hacen difícil para los estudiantes universitarios mantener hábitos alimenticios saludables.

Otro factor que también entro en la ecuación es el confinamiento que se está viviendo actualmente debido a la pandemia del COVID-19. Aquí es donde surge la pregunta, mejoraron o empeoraron los hábitos alimenticios durante el confinamiento.

Para responder a esa pregunta nos basamos en el Test Kimed, de acuerdo al índice de alimentación saludable de la población española que consta de 16 ítems, adaptado a los estudiantes del Estado de Coahuila, ya que es una prueba basada en la dieta mediterránea que es considerada de las mejores a nivel mundial y está caracterizada por un alto consumo de verduras, frutas y hortalizas, legumbres, frutos secos, cereales y aceite de oliva; junto con un consumo moderado de pescados, huevos y productos lácteos, preferentemente yogur o queso, y un menor consumo de carnes y grasas animales. La dieta mediterránea no solo garantiza un aporte calórico y de nutrientes en cantidades suficientes y proporciones adecuadas, sino que, además, contribuye a la prevención de enfermedades cardiovasculares, diabetes, cáncer, enfermedades degenerativas, etc. y, en general, a una mayor esperanza de vida (Durá \& Castroviejo, 2011, p. 603).

En México la dieta y la alimentación se han estudiado sobre todo mediante las Encuestas Nacionales de Nutrición y Salud, que se centran en el análisis del consumo de alimentos. Sin embargo, la agrupación de los alimentos que se emplean para evaluar los resultados de estas encuestas es complicado, por ejemplo, dan el mismo valor al pan dulce y las donas que a las tortillas de maíz, o a los tamales de maíz que a la mayonesa. Desde esas interpretaciones no se consideran las funciones de los componentes bioactivos por lo que no se puede distinguir entre patrones de consumo tradicional o moderno. (Muñoz, et.al.,2015, p. 1583)

La Secretaría de Salud, el Instituto Nacional de Salud Pública(INSP) y el Instituto Nacional de Estadística y Geografía (INEGI), realizaron la Encuesta Nacional de Salud y Nutrición (ENSANUT) 2018, con el objeto de conocer el estado de salud y las condiciones nutricionales de la población en México. Los resultados principales fueron:

Consumo de alimentos recomendables

\begin{tabular}{ccccccc}
\hline Alimentos & Lácteos & Frutas & Leguminosas & Carnes & Huevo & Verduras \\
\hline $12-19$ años & $37 \%$ & $35.2 \%$ & $37 \%$ & $50 \%$ & $28.9 \%$ & $24.9 \%$ \\
\hline 20 años o más & $47 \%$ & $49.7 \%$ & $54.1 \%$ & $64.6 \%$ & $29.9 \%$ & $44.9 \%$ \\
\hline
\end{tabular}

Consumo de alimentos no recomendables

\begin{tabular}{lllllll}
\hline Alimentos & $\begin{array}{c}\text { Bebidas } \\
\text { no lácteas- } \\
\text { endulzadas }\end{array}$ & $\begin{array}{c}\text { Botanas, } \\
\text { dulces y } \\
\text { postres }\end{array}$ & $\begin{array}{c}\text { Cereales } \\
\text { dulces }\end{array}$ & $\begin{array}{c}\text { Bebidas } \\
\text { lácteas } \\
\text { endulzadas }\end{array}$ & $\begin{array}{c}\text { Comida } \\
\text { rápida y } \\
\text { antojitos } \\
\text { mexicanos }\end{array}$ & $\begin{array}{c}\text { Carnes } \\
\text { procesadas }\end{array}$ \\
\hline 12 -19 años & $85.7 \%$ & $53.7 \%$ & $35.2 \%$ & $10.9 \%$ & $22.9 \%$ & $10.2 \%$ \\
\hline 20 años o más & $85.8 \%$ & $35.4 \%$ & $33.9 \%$ & $16.8 \%$ & $20.3 \%$ & $7.4 \%$ \\
\hline
\end{tabular}


En el rango de edad de 12 a 19 años el 39.7\% de la población tiene sobrepeso y obesidad, en el área urbana. En el rango de 20 años o más, el 76.8\% de mujeres tiene sobrepeso y obesidad y $73 \%$ de hombres. En Coahuila el $16.6 \%$ de la población tiene sobrepeso y obesidad.

En base a la información recabada se plantean las siguientes Hipótesis:

H0: No existe diferencia significativa entre estudiantes universitarios hombres y mujeres y sus hábitos de alimentación saludable

H1: Si existe diferencia significativa entre estudiantes universitarios hombres y mujeres y sus hábitos de alimentación saludable

H0: No existe diferencia significativa entre los rangos de edad de los estudiantes universitarios y sus hábitos de alimentación saludable

H2: Si existe diferencia significativa entre los rangos de edad de los estudiantes universitarios y sus hábitos de alimentación saludable

\section{MÉTODO}

Esta investigación presenta un análisis explicativo, transversal, no experimental, y cuantitativo. Aplicando un nivel de confianza del 95\% y un margen de error del 5\%, se obtuvo un tamaño de muestra de 408 personas. Se aplicó en los meses de febrero y marzo de 2021 a jóvenes estudiantes universitarios del estado de Coahuila, de las áreas de ciencias económico-administrativas, ciencias sociales, ingenierías, ciencias de la salud y diseño. El análisis de información se hizo mediante el paquete estadístico SPSS versión 23. Se realizaron pruebas no paramétricas de Mann-Withney y de Kruskal-Wallis para probar las hipótesis.

Se aplicó el test Kidmed, de acuerdo al índice de alimentación saludable de la población española que consta de 16 ítems que deben responderse de manera afirmativa/negativa (si/no). Las respuestas afirmativas en las preguntas que representan un aspecto positivo en relación con una alimentación saludable (son 12) suman 1 punto, y las respuestas afirmativas en las preguntas que representan una connotación negativa en relación a una alimentación saludable (son 4) restan 1 punto. La puntuación total obtenida da lugar al índice Kidmed, que se clasifica en tres categorías: a) De 8 a 12: alimentación saludable óptima b) De 4 a 7: necesidad de mejorar los hábitos alimentarios (media). c) De 0 a 3: dieta de muy baja calidad.

En esta investigación se aplicaron los 16 ítems antes de la pandemia y durante la pandemia, resultando 32 ítems. También se preguntaron variables sociodemográficas: sexo, edad, carrera, con quien vivían y su percepción en cuanto a los cambios de hábitos alimentarios a raíz de la pandemia de Covid-1 9.

\section{HALLAZGOS}

Los resultados obtenidos de la investigación son: las variables sociodemográficas utilizadas fueron: de un total de 408 estudiantes universitarios 54.4\% son mujeres y $45.6 \%$ hombres. Los rangos de edad fueron: $24.5 \%$ de 18 a 19 años; $40 \%$ de 20 a $21 ; 15.2 \%$ de 22 a 23; $4.9 \%$ de 24 a 25 y $13.5 \%$ mayores de 25 años. La distribución de las carreras fue: $42.2 \%$ de ciencias económico-administrativas; $28.4 \%$ ingenierías; $13 \%$ ciencias de la salud; $11.8 \%$ ciencias sociales y $4.7 \%$ diseño. Con quien vives se obtuvo que el $87.8 \%$ vive con familiares (padre, madre, hermanos), 2.4\% con compañeros o amigos, 
$1.7 \%$ solos y $8.1 \%$ con pareja. Cabe mencionar que por la pandemia del Covid-19, muchos estudiantes regresaron al hogar paterno.

La tabla 1 también muestra la comparación de los hábitos alimenticios antes y durante la pandemia de Covid-19 y se encontró que aumentaron los porcentajes de los buenos hábitos en general, y en particular el de las mujeres en cuanto al consumo de frutas o jugos naturales, así como comer una segunda fruta todos los días, consumir verduras frescas más de una vez al día y aumentó el consumo de pescado. También disminuyeron los hábitos negativos como consumir comida rápida, desayunar bollería industrial y consumir golosinas varias veces al día.

Tabla 1. Tabla cruzada de consumo de alimentos saludables/género

\begin{tabular}{|c|c|c|c|c|}
\hline VARIABLES & $\begin{array}{l}\text { HOMBRE } \\
\text { Antes } \\
\text { Pandemia }\end{array}$ & $\begin{array}{l}\text { MUJERES } \\
\text { Antes } \\
\text { Pandemia }\end{array}$ & $\begin{array}{l}\text { HOMBRES } \\
\text { Durante } \\
\text { Pandemia }\end{array}$ & $\begin{array}{c}\text { MUJERES } \\
\text { Durante } \\
\text { Pandemia }\end{array}$ \\
\hline $\begin{array}{l}\text { 1-Consumes una fruta o un jugo natural } \\
\text { todos los días }\end{array}$ & $61.3 \%$ & $61.3 \%$ & $60.2 \%$ & $68.0 \%$ \\
\hline $\begin{array}{l}\text { 2-Consumes una segunda pieza de fruta } \\
\text { todos los días }\end{array}$ & $29.6 \%$ & $40.1 \%$ & $34.4 \%$ & $46.8 \%$ \\
\hline $\begin{array}{l}\text { 3-Consumes verduras frescas (ensaladas) } \\
\text { o cocinadas, regularmente una vez al } \\
\text { día. }\end{array}$ & $64 \%$ & $77 \%$ & $66.1 \%$ & $81.1 \%$ \\
\hline $\begin{array}{l}\text { 4-Consumes verduras frescas o } \\
\text { cocinadas de forma regular más de una } \\
\text { vez al día. }\end{array}$ & $46.8 \%$ & $63.1 \%$ & $52.7 \%$ & $69.4 \%$ \\
\hline $\begin{array}{l}\text { 5-Consumes pescado con regularidad } \\
\text { (por los menos 2-3 veces a la semana). }\end{array}$ & $25.8 \%$ & $28.4 \%$ & $22.6 \%$ & $33.8 \%$ \\
\hline $\begin{array}{l}\text { 6-Acude una vez o más a la semana a } \\
\text { un centro de comida rápida (fast food) } \\
\text { (hamburguesería o pizzería.) }\end{array}$ & $62.9 \%$ & $67.6 \%$ & $53.8 \%$ & $48.6 \%$ \\
\hline $\begin{array}{l}\text { 7-Consume legumbres (frijol, garbanzo, } \\
\text { habas, chicharos, etc.) más de } 1 \text { vez a la } \\
\text { semana. }\end{array}$ & $91.4 \%$ & $92.8 \%$ & $93.0 \%$ & $94.1 \%$ \\
\hline $\begin{array}{l}\text { 8-Consume pasta o arroz casi a diario ( } 5 \\
\text { días o más a la semana) }\end{array}$ & $64.5 \%$ & $76.6 \%$ & $64.5 \%$ & $76.6 \%$ \\
\hline $\begin{array}{l}\text { 9-Desayuna un cereal o derivado (pan, } \\
\text { etc.) }\end{array}$ & $64.0 \%$ & $68.5 \%$ & $63.4 \%$ & $67.6 \%$ \\
\hline $\begin{array}{l}\text { 10-Consume frutos secos (nueces, } \\
\text { almendras, cacahuates, avellanas, etc.) } \\
\text { con regularidad (al menos 2-3 veces a la } \\
\text { semana). }\end{array}$ & $54.8 \%$ & $53.2 \%$ & $55.9 \%$ & $59.5 \%$ \\
\hline $\begin{array}{l}\text { 11-Utiliza aceite de oliva en casa para } \\
\text { cocinar. }\end{array}$ & $49.5 \%$ & $44.1 \%$ & $50.0 \%$ & $49.5 \%$ \\
\hline 12-No desayuna todos los días. & $48.4 \%$ & $47.3 \%$ & $44.1 \%$ & $49.5 \%$ \\
\hline $\begin{array}{l}\text { 13-Desayuna un lácteo (yogurt, leche, } \\
\text { etc.) }\end{array}$ & $68.3 \%$ & $69.8 \%$ & $64.5 \%$ & $61.7 \%$ \\
\hline
\end{tabular}




\begin{tabular}{llllll}
\hline $\begin{array}{l}\text { 14-Desayuna bollería } \\
\text { galletas, pan o pastelitos. }\end{array}$ & industrial, & $58.1 \%$ & $50.0 \%$ & $49.5 \%$ & $41.0 \%$ \\
\hline $\begin{array}{l}\text { 15-Consume 2 yogures y/o } 40 \mathrm{~g} \text { de } \\
\text { queso cada día. }\end{array}$ & $28.0 \%$ & $30.6 \%$ & $25.8 \%$ & $28.4 \%$ \\
\hline $\begin{array}{l}\text { 16-Consume golosinas y/o caramelos } \\
\text { varias veces al día. }\end{array}$ & $46.8 \%$ & $55.0 \%$ & $38.2 \%$ & $47.7 \%$ \\
\hline
\end{tabular}

Elaboración propia

Para la comprobación de las hipótesis, se realizó un estudio de pruebas no paramétricas. Se realizó una prueba de Mann-Withney para dos muestras independientes, en el caso de la variable género y resultó que si existe diferencia significativa entre hombres y mujeres con una significancia menor a 0.05 , en las siguientes variables (Tabla 2). Como se puede observar en la tabla 2 fueron las mismas variables que tuvieron diferencia significativa a excepción de que durante la pandemia se agregó una variable, respecto al consumo de pescado.

En todas las variables significativas, las mujeres tienen un mayor consumo tanto de frutas, como de verduras, arroz y pescado. Otro factor a considerar es que las mujeres tienen mayor tendencia a cuidar la figura y hacer dietas.

Tabla 2. Prueba de Mann-Withney

\begin{tabular}{lclcc}
\hline \multicolumn{1}{c}{$\begin{array}{c}\text { Variables } \\
\text { Antes de la pandemia }\end{array}$} & $\begin{array}{c}\text { Significancia } \\
\text { asintótica } \\
\text { (bilateral) }\end{array}$ & \multicolumn{1}{c}{$\begin{array}{c}\text { Vuriables. } \\
\text { Durante la pandemia }\end{array}$} & $\begin{array}{c}\text { Significancia } \\
\text { asintótica } \\
\text { (bilateral) }\end{array}$ \\
\hline $\begin{array}{l}\text { 2-Consumes una segunda pieza } \\
\text { de fruta todos los días }\end{array}$ & 0.027 & $\begin{array}{l}\text { 2-Consumes una segunda } \\
\text { pieza de fruta todos los días }\end{array}$ & 0.011 \\
\hline $\begin{array}{l}\text { 3-Consumes verduras frescas } \\
\text { (ensaladas) o cocinadas, } \\
\text { regularmente una vez al día }\end{array}$ & 0.004 & $\begin{array}{l}\text { 3-Consumes verduras frescas } \\
\text { (ensaladas) o cocinadas, } \\
\text { regularmente una vez al día }\end{array}$ & 0.001 \\
\hline $\begin{array}{l}\text { 4-Consumes verduras frescas o } \\
\text { cocinadas de forma regular más } \\
\text { de una vez al día }\end{array}$ & 0.001 & $\begin{array}{l}\text { 4-Consumes verduras frescas } \\
\text { o cocinadas de forma regular } \\
\text { más de una vez al día. }\end{array}$ & 0.001 \\
\hline $\begin{array}{l}\text { 8-Consume pasta o arroz casi a } \\
\text { diario (5 días o más a la semana) }\end{array}$ & 0.008 & $\begin{array}{l}\text { 8-Consume pasta o arroz casi } \\
\text { a diario (5 días o más a la } \\
\text { semana) }\end{array}$ & 0.008 \\
\hline & & $\begin{array}{l}\text { 5-Consumes pescado con } \\
\text { regularidad (por los menos 2-3 } \\
\text { veces a la semana). }\end{array}$ & 0.013 \\
\hline
\end{tabular}

Se realizó una prueba de Kruskal-Wallis para muestras independientes con la variable edad, resultando que si hay diferencia significativa entre los diferentes rangos de edad (tabla 3), específicamente en 5 variables: consumo de pescado, legumbres, frutos secos, comida rápida y golosinas. Como puede observarse 3 hábitos positivos y 2 negativos. 
Tabla 3. Prueba de Kruskal-Wallis

\begin{tabular}{lccc}
\hline Variables Antes de la pandemia & $\begin{array}{c}\text { Significancia } \\
\text { asintótica } \\
\text { (bilateral) }\end{array}$ & $\begin{array}{c}\text { Variables. Durante la } \\
\text { pandemia }\end{array}$ & $\begin{array}{c}\text { Significancia } \\
\text { asintótica } \\
\text { (bilateral) }\end{array}$ \\
\hline $\begin{array}{l}\text { 5-Consumes pescado con } \\
\text { regularidad (por los menos } 2-3\end{array}$ & 0.036 & \\
\begin{tabular}{l} 
veces a la semana). \\
\hline
\end{tabular}
\end{tabular}

6-Acude una vez o más a la semana a un centro de comida rápida (fast food) (hamburguesería o pizzería.)

7-Consume legumbres (frijol, garbanzo, habas, chicharos, etc.) $\quad 0.039$ más de 1 vez a la semana.

10-Consume frutos secos (nueces, almendras, cacahuates, avellanas, etc.) con regularidad (al menos 2-3 0.047 (nueces, almendras, cacahuates, veces a la semana).

10-Consume frutos secos avellanas, etc.) con regularidad 0.020 (al menos 2-3 veces a la semana).

$\begin{array}{lll}\begin{array}{l}\text { 16-Consume golosinas } \\ \text { caramelos varias veces al día }\end{array} & \text { y/o } & 0.011\end{array}$

Los hábitos de consumo de alimentos, antes de la pandemia, que resultaron significativos fueron: consumo de pescado, legumbres y de frutos secos, los practican estudiantes con 24 años o más. Sin embargo, los estudiantes que también practican 2 hábitos negativos como son consumir comida rápida y consumir golosinas también son los que tienen 24 a 25 años. Durante la pandemia solo una variable presento diferencia significativa, el consumo de frutos secos siendo el rango de edad que más los consume de 20 a 21 años.

En base a los resultados obtenidos en general, durante la pandemia los estudiantes que tienen hábitos positivos como son: consumir fruta, verduras, pescado, legumbres, frutos secos y desayunar lácteos, predominan los estudiantes en un rango de edad de 20 a 23 y mayores de 25 años. Por otra parte, los hábitos negativos como son: consumir comida rápida, no desayunar, desayunar bollería industrial y consumir golosinas se presenta en el rango de edad de 24 a 25 años.

Respecto al resultado en base a la aplicación del Test Kidmed, se encontró que antes de la pandemia se obtuvo un puntaje de 8 hábitos positivos y 3 negativos quedando como resultado un índice de 5, lo que equivale a la categoría media, (4 a 7): necesidad de mejorar los hábitos alimentarios.

Durante la pandemia cambió el panorama, se obtuvieron los mismos 8 hábitos positivos, pero solo uno negativo, quedando un índice de 7, lo que equivale a la categoría de media, (4 a 7): necesidad de mejorar los hábitos alimentarios.

Aunque mejoro la calificación siguieron quedando en una alimentación media, donde es necesario mejorar la dieta que actualmente consumen. 


\section{DISCUSIÓN/CONCLUSIONES}

Se analizaron las respuestas de 408 estudiantes de educación superior de diferentes universidades en el Estado de Coahuila, sobre su régimen de alimentación antes y durante la pandemia de Covid-19, para determinar si su dieta incluía alimentos saludables (12 variables) y si practicaban hábitos nocivos para su salud (4variables). Se encontró que antes de la pandemia, el 56.6\% de la muestra de estudiantes tenía hábitos positivos de alimentación como son: consumir fruta una vez al día, consumir verduras una vez al día, consumir legumbres, desayunar cereal, consumir frutos secos, utilizar aceite de oliva, desayunar lácteos y algunos consumir pescado. Pero no solo practicaban buenos hábitos sino también malos hábitos como: acudir una vez o más a la semana a un centro de comida rápida (fast food), no desayunar o si lo hace, desayunar bollería industrial (galletas, pastelitos o pan) o consumir golosinas y/o caramelos varias veces al día. Realmente sus hábitos alimenticios no eran buenos antes de la pandemia y como otros estudios lo han demostrado las causas son varias. La mala alimentación es frecuente en los alumnos universitarios. Una gran cantidad de estudios ha mencionado esta situación, identificando como características alimentarias el consumo de alimentos de alta densidad energética y limitando la ingesta de alimentos como frutas y verduras, en donde la carrera que cursan no haría diferencia frente a esta situación. (Troncoso, et.al., 2013, p.45) Así como lo mencionan los autores anteriores, hay estudios que realizados por diferentes investigadores donde concluyen que el conocimiento que se tenga de una alimentación saludable no es un factor decisivo para ponerla en práctica ya que se han encontrado otros factores que se convierten en determinantes a la hora de elegir que alimentos comprar como son: el costo de los alimentos, la falta de oferta de alimentos saludables en las cafeterías o puestos cercanos a la universidad o dentro del área de la misma y la limitante de tiempo disponible entre clases, actividades y trabajo. En la investigación realizada por Troncoso et al., encontraron que los estudiantes de pedagogía identifican con claridad que deben realizar una alimentación que codifican como sana, pero que en la práctica no es seguida por ellos, debido a diversas circunstancias como los costos de alimentos, la falta de oferta de alimentos en el centro educacional y la inadecuada selección de estos últimos que los estudiantes realizan. $(2013$, p.45)

Respecto a la primera hipótesis se rechaza ya que se encontró que, si existe diferencia significativa entre hombres y mujeres, en cuanto a sus hábitos alimenticios. Así como lo menciona Rodríguez (2015), en su estudio encontró que las mujeres tienen mejores hábitos de alimentación que los hombres, las mujeres tienen mayor conocimiento que los varones sobre alimentación saludable y la ingesta de verduras. Mencionado por Reyes y Oyola (2020, p.71).

Respecto a la segunda hipótesis se rechazó ya que si hay diferencias significativas entre las edades de los estudiantes respecto a sus hábitos de alimentación saludable. En cuanto a la edad el resultado fue que el rango de edad que mejores hábitos alimenticios tienen son estudiantes de 20 a 23 años. Los hábitos negativos se presentaron más en los estudiantes de 24 a 25 años. Es muy probable que los de mayor edad la mayoría ya trabajan y tienen que equilibrar el tiempo entre actividades académicas y trabajo y eso les disminuye mucho el tiempo que le dedican a buscar una oferta sana de alimentos que se encuentre cerca ya sea de la universidad o de su trabajo. Así lo demostró Gonzales (2018) en el estudio con estudiantes universitarios que tienen un consumo elevado de azúcares y horarios inadecuados de alimentación. Mencionado por Reyes y Oyola (2020, p.71).

Respecto a los resultados del test Kidmed, tanto antes como durante la pandemia los estudiantes universitarios presentan un nivel medio de alimentación, lo que significa que tienen necesidad de mejorar los hábitos alimentarios. Pasaron de 5 a 7 en el índice. 
Aún a pesar de haber mejorado en un $2 \%$ los hábitos de alimentación de los estudiantes universitarios durante el confinamiento de la pandemia de Covid-19, sobresaliendo el aumento en el consumo de frutas, verduras frescas, legumbres, frutos secos y pescado. Así mismo disminuyo el porcentaje de estudiantes que consumen comida rápida, que no desayuna, que desayunan bollería industrial y que consumen golosinas. Lo más probable es que se deba a que el 88\% vive con familiares (padres y hermanos) y los foráneos regresaron a sus casas, lo que significa que están bajo la supervisión paterna, también aumento la preparación de comidas dentro del hogar y esto ayudó a mejorar su alimentación.

Si bien es cierto aún no se logra que tengan una alimentación optima, el porcentaje de estudiantes con buenos hábitos alimenticios es de un 56\%, se pudo observar que al menos se reforzaron hábitos positivos y se disminuyó en hábitos negativos, sin embargo, falta mucho por hacer en cuanto a educar a este segmento de la población, teniendo una ventana de oportunidad para las universidades para educar y concientizar a los estudiantes en mejorar sus hábitos alimenticios y ayudar a disminuir enfermedades no trasmisibles como la hipertensión, diabetes y obesidad.

Una de las limitaciones del estudio es la falta de valores de peso y altura, para evaluar el estado nutricional de los estudiantes, también es recomendable considerar otras variables como el nivel socioeconómico, cambio de patrones de consumo por el uso de plataformas para pedir a domicilio, el consumo de alimentos no saludables por el estrés del confinamiento y el impacto de la publicidad.

\section{REFERENCIAS}

1. Agenda de Salud Sostenible para las Américas 2018-2030 (CSP29/6)

2. Durá, T. y Castroviejo, A. (2011) Adherencia a la dieta mediterránea en la población universitaria. Nutrición Hospitalaria,26(3),602-608. doi:10.3305/nh.2011.26.3.4891

3. ENSANUT (2018) Encuesta Nacional de Salud y Nutrición

4. FINUT (2018) Fundación Iberoamericana de Nutrición

5. Gaona, EB., Martínez, B., Arango, A., Valenzuela, D., Gómez, L., Shamah, T., Rodríguez, S.

6. (2018). Consumo de grupos de alimentos y factores sociodemográficos en población mexicana. Salud Pública de México, 60(3), 272-282. https://doi.org/10.21149/8803

7. González, M., Castañeda, D., De León, A., Rivas, F. (2018) Study on eatinghabits in a student

8. population of the Faculty of Engineering of the Autonomous University of San Luis Potosí. Revista de resultados negativos y no positivos. 3(2) 86-158.

9. INEGI. (2017) Concentrado Entidad del Sistema Educativo Coahuilense. Ciclo Escolar 2017-2018

10. Martínez, M., Hernández, D., Ojeda, M., Mena, R., Alegre, A., y Alfonso, J. (2009) Desarrollo de un programa de educación nutricional y valoración del cambio de hábitos alimentarios saludables en una población de estudiantes de Enseñanza Secundaria Obligatoria. Nutrición Hospitalaria, 24(4), 504-510

11. Martínez, I \& Villezca, P. (2005) La alimentación en México un estudio a partir de la encuesta nacional de ingresos y gastos de los hogares y de las hojas de balance alimenticio de la FAO. Ciencia UANL, 8(1), 196-208.

12. Mc Coll C, Amador C, Aros B, Lastra C, Pizarro S. (2002) Prevalence of risk factors for chronic noncommunicable diseases in medical students of the University of Valparaíso. Rev. Chil. Pediatr. 73 (5), 478-482. http://dx.doi.org/10.4067/S0370-4106200200050000510.

13. Muñoz J, Córdova, J y Del Valle, D. (2015). El índice de alimentación saludable de estudiantes de nuevo ingreso a una universidad de México. Nutrición Hospitalaria, 31(4):1582-1588. doi:10.3305/nh.2015.31.4.8401

14. Navarro, I., Ros, G., Martínez, B., Rodríguez, A., Periago, M. (2016). Adherencia a la dieta mediterránea y su relación con la calidad del desayuno en estudiantes de la Universidad de Murcia. Nutrición Hospitalaria, 33(4), 901-908 doi:http://dx.doi.org/10.20960/nh.390 
15. Navarro, S., González, E., Montero, M., López, M., Schmidt, J. (2015). Estilo de vida y seguimiento de la ingesta dietética en estudiantes del Campus de la Universidad de Granada en Melilla. Nutrición Hospitalaria, 31(6), 2651-2659. doi:10.3305/nh.2015.31.6.8973

16. Organización Panamericana de la Salud (OPS). https://www.paho.org/es/agenda-saludsosteniblepara-americas-2018-2030. Recuperado el 09de marzo de 2021.

17. Pérez, L., Bayona, I., Benito, M. (2007). Test e índice KIDMED en cinco grupos de estudiantes

18. europeos Rev Esp Nutr Comunitaria,13(3-4), 124-129

19. Pi, R., Vidal, P., Brassesco, B., Viola, L. and Aballay, L. (2015) Nutritional status in university

20. students: its relationship with the number of daily food intakes and the consumption of macronutrients. Nutrición Hospitalaria, 31(4), 1748-1756.

21. Reyes, S., Oyola, M. (2020) Conocimientos sobre alimentación saludable en estudiantes de una

22. universidad pública. Rev Chil Nutr, 47(1), 67-72. http://dx.doi.org/10.4067/S071775182020000100067

23. Rodríguez H, Restrepo L, Deossa G. (2015). Knowledge and practices on nutrition, health and

24. exercise in university students of Medellín-Colombia. Perspect Nut Hum, 17(1), 36-54.

25. Sánchez V, Aguilar A, González F, Esquius L, Vaqué C. (2017) Evolution in knowledge about

26. food: an educational intervention in university students. Rev Chil Nutr, 44(1), 19-27

27. Troncoso C, Doepking C, Zúñiga C. (2013). Healthy eating in the training of career students in the

28. pedagogical area. Rev Chil Nutr, 40(1), 43-47.

29. Vivas, R. (2020) Patrones de consumo en la alimentación de los individuos de altos ingresos de Bogotá, ante la pandemia COVID-19. (tesis de pregrado). Colegio de Estudios Superiores de Administración de Empresas, Bogotá, Colombia.

30. World Health Organization. WHO. Healthy food. Geneva. 2015.

31. World Health Organization. WHO. Healthy food. 2018. [Accessed marzo 18, 2021]. Available in: https:/www.who.int/es/news-room/fact-sheets/detail/obesity-and-overweight 